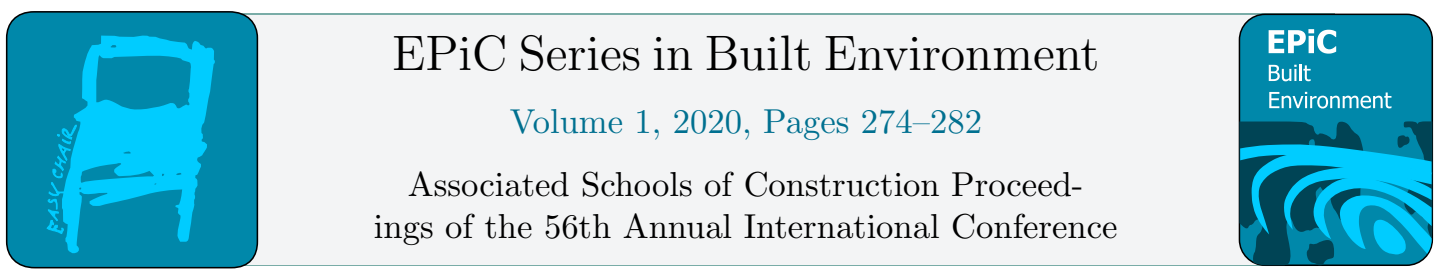

\title{
Blockchain-based Information Sharing: A New Opportunity for Construction Supply Chains
}

\author{
Pardis Pishdad-Bozorgi, Ph.D., Jong Han Yoon, Ph.D. Student, Nishant Dass, Ph.D. \\ Georgia Institute of Technology \\ Atlanta, Georgia
}

In the supply chain, information sharing is critical for facilitating decision making, accelerating the schedule, reducing costs, and enhancing the final quality of the project. However, information sharing is challenging in the construction supply chain when information asymmetry could help certain individuals avoid liability or help others maximize their profits by using substandard materials. In this environment, the stakeholders may not truly trust one another and may be reluctant to share complete information. Even though several studies have emphasized the importance of trust for information sharing, few studies have focused on promoting trust and verifying information using technology. The purpose of this paper is to examine the potential of Blockchain technology for establishing a trust-enhanced environment with distributed trust that will benefit the construction supply chain. This paper presents three logical scenarios for the application of Blockchain technology in information-sharing systems in order to demonstrate that the technology helps establish a trust-enhanced environment for reliable information sharing while eliminating counterfeiting and reducing bullwhip effect. These scenarios will demonstrate the value of Blockchain-based information sharing in the construction supply chain and possibly provide direction for future research with greater depth and specificity.

Key Words: Blockchain, Construction Supply Chain Management, Information Sharing, Trust

\section{Introduction}

Construction supply chain management (CSCM) facilitates cooperation and collaboration between suppliers by streamlining the flow of money, product, and information in order to promote the efficiency and effectiveness of the construction supply chain (CSC). The information flow in a supply chain is critical for facilitating decision making, accelerating the schedule, reducing costs, and enhancing the final quality of the project (Cheng et al., 2010; Deng et al., 2019; Kim \& Chai, 2017). This is because the information flow directly impacts the production scheduling, inventory control, and delivery plans of individual members. Promoting information sharing is one of the most impactful methods to improve supply chain efficiency and effectiveness. Construction supply chains often have information flow that is not well integrated because the information may be fragmented in various stages or across numerous stakeholders (e.g., owners, Architects/Engineers (A/E), General Contractors (GC), Sub-Contractors (Subs), and raw material suppliers) (Fischer et al., 2017). This poses a significant challenge to the information sharing and negatively impacts the cost, time, and quality of projects (Cheng et al., 2010). In order to resolve this problem, many studies have tried to 
develop information-sharing platforms with technologies such as BIM, GIS, GPS, and RFID (Deng et al., 2019; Irizarry et al., 2013; Shin et al., 2011; Wang et al., 2007). However, despite the many attempts to develop an information-sharing platform, the lack of information integration is still a problem in the construction industry. The reason why the information sharing is still challenging is the lack of trust and absence of a guarantee for data privacy. The presence of trust-based relationships strongly facilitates information sharing (Jiang et al., 2012). However, in a construction project, each stakeholder may have opportunistic behavior and seek their individual profits through information asymmetry caused by the lack of integration (Xiang et al., 2012). In such scenarios, the stakeholders do not trust one another and thus consider it better to take the opportunistic approach. In the presence of trust, if the parties realize that they are better off once they collaborate and share information with their partners, they choose to be more cooperative and open to information sharing. Unfortunately, little attention has been paid to construction suppliers' relationship management (CSRM), which aims to establish or promote trust between stakeholders. This study intends to illustrate how trust is established between construction suppliers using Blockchain technology. In terms of methodology, this study first conducts literature reviews regarding CSCM and Blockchain applications in the construction industry. Based on the reviews, the authors introduce three scenarios that discuss the CSC-specific problems that are addressable with a Blockchain-based information sharing platform. Secondly, through the expert interviews with experts in Blockchain and construction industry, the authors verify the technical feasibility of the ideas discussed in the three scenarios and validate the technology's potential effectiveness.

\section{What is Missing in Construction Supply Chain Management?}

The fragmented nature of CSC leads to low productivity, cost and time overruns due to change orders, inadequate design specifications, liability claims, conflicts and disputes, etc. (Behera et al., 2015). To address this issue, CSCM has focused on collaboration and information sharing for effective material management and asset flow throughout construction supply chain (Shin et al., 2011). This focus has promoted the development of information-sharing platform to reduce logistics costs, duration of site activities, lead time and inventory by integrating technologies such as BIM, GIS, GPS, and RFID. The developed platforms have mostly focused on the supply chain between GC and Subs, but there is no guarantee that the suppliers will share their information. Suppliers' relationship management closely affects the suppliers' willingness to share information throughout supply chains. Trust-based relationships among suppliers increase their willingness for sharing information and encourage the stakeholders' commitments to project values (Jiang et al., 2012). Thus, the relationships should be managed in terms of trust. However, the research on a systematic solution that promotes trust through the application of technology has been in its infancy, compared to the studies focusing on system development for optimizing logistics in CSC.

\section{Literature on Construction Supply Chain Management}

Several previous studies have emphasized information sharing and provided ways to share information using a novel system (Cheng et al., 2010; Deng et al., 2019; Irizarry et al., 2013; Nummelin et al., 2011; Shin et al., 2011; Wang et al., 2007). When stakeholders (e.g., owners, A/E, GCs, Subs, Suppliers, and Manufacturers) in CSC could share information without difficulty, some of the current problems leading to underperformance would be solved. For example, information-sharing can solve change order abuse or counterfeited materials by eliminating information asymmetries. Most studies on CSCM have just focused on optimizing logistics between GC to Subs with real-time information sharing (Cheng et al., 2010; Deng et al., 2019; Irizarry et al., 2013; Shin et al., 2011; Wang et al., 2007), however information-sharing is critical to all stakeholders in the supply chain. 
Although the studies addressing BIM include the information sharing among all stakeholders, the shared information is just limited to optimizing logistics (Nummelin et al., 2011, being an exception). Additional studies are needed on both information sharing among all stakeholders and how they can utilize the shared information for increasing project value. Furthermore, establishing trust between suppliers should be addressed as well. For the suppliers to willingly share their information, relationship management for establishing and promoting trust is necessary.

\section{Literature on Construction Suppliers' Relationship Management Focusing on Trust}

A few studies on CSRM have focused on trust. These studies have emphasized the importance of trust for CSC integration. In particular, Jiang et al. (2012) and Saini et al. (2019) discuss how trust encourages suppliers to share information. While there has been constant emphasis on the positive impact of trust-based relationship among suppliers, few systematic solutions exist that promote trust using technology beyond what people could achieve in terms of establishing personal trust. Meng (2010) developed a systematic model for the assessment of CSC relationships. In this model, Meng (2010) pointed out that trust is one of the most important indicators to estimate suppliers' relationship, which impacts performance of the supply chain. Ryciuk (2017) proposes indicators for estimating trust in CSC. He argues that a high level of trust between supply chain members can improve CSC by increasing the speed with which decisions are made and reducing costs of implementation. Various types of information-sharing platforms have been developed, but their full implementation has not been realized and that itself might be due to the lack of trust among stakeholders. The full use of these systems can come to fruition only when enough trust generates the willingness among the stakeholders to share their information. If systematic methods providing a trust-enhanced environment are developed and leveraged in conjunction with the information-sharing platforms, we can synergistically impact information integration in CSC, and thus lower project costs, save the environment, enhance quality of projects, and shorten the project schedule.

\section{Why Blockchain Technology?}

Blockchain technology can provide a trust-enhanced environment across all members of a CSC as opposed to any one entity. Blockchain is an immutable and decentralized digital ledger that contains linked blocks including transaction data (Samaniego \& Deters, 2016). The data in the block are protected using encryption via a hash function and the blocks are linked to each other with the hash value, which is the cryptographic value made by the data stored in the block. When new blocks get added to the chain, they must satisfy the criteria of the "consensus protocol" such as Proof-of-Work or Proof-of Stake (Mougayar, 2016). The blockchain is replicated and distributed to every participant node, thus creating a decentralized ledger (Lisk, 2019). Through these capabilities, the data cannot be falsified or tampered with. In this environment, the participants are able to trust the reliability and security of the data. Furthermore, Blockchain is capable of executing a smart contract, which is a computer protocol intended to digitally facilitate, verify, or enforce the negotiation or performance of a contract (Rosic, 2016). Smart contracts provide the rules and penalties with an automatic transaction process and thus facilitates the fulfillment of obligations without human intervention (Rosic, 2016).

Smart contracts will encourage participants to be committed to the contract conditions while preventing opportunistic behavior such as counterfeiting. The immutability, decentralization and smart contract features of Blockchain promote trustworthiness in information sharing regarding quality, quantity, timeliness, and cost of products.

Despite the benefits of Blockchain, several challenges regarding applying it to businesses remain. Even though the trusted intermediaries are fully replaced by distributed trust, the consensus protocol 
of Blockchain is demanding, making the speed of transactions slow for many businesses. In addition, Businesses are skeptical of putting their trade secrets and sensitive data on a public Blockchain where literally anyone can participate. If public Blockchains are an anathema to businesses, then "private, permissioned Blockchains" may be the answer. Private Blockchains overcome the two main criticisms of public blockchains - they neither suffer from slow transaction speeds nor are they based on networks that are open to just about anybody. Even though the private Blockchain enables businesses to apply Blockchain in a permissioned environment, Blockchain is difficult to implement - it is both technologically complex and hard to find the right talent for building it. Furthermore, a distributed ledger only makes sense when the network is large because of two reasons for it: economic reason network effects grow exponentially with the size of the network; and technical reason - a smaller network will be vulnerable to a $51 \%$ attack. As we can see from the limitations, Blockchain technology is still in its infancy. However, note that the internet was invented in the early-1990s and it has taken 25 years for it to reach mass adoption; whereas it has only been 10 years since the first Bitcoin started trading, so by some measure, there is still another 10 or more years before Blockchain reaches mass adoption. Nonetheless, we argue that the technology holds tremendous potential for benefitting the construction industry.

\section{Literature on Blockchain applications in the construction industry}

In recent years, several papers have pointed out the potential use of Blockchain to improve the construction industry. Barima (2017) and Belle (2017) examined the potential use of Blockchain with several scenarios in the construction industry. Barima (2017) focused on improving the construction procurement process by using Blockchain, and Belle (2017) emphasized the Blockchain-based digital infrastructure, which enables storing and tracing data transactions, for the Architecture, Engineering, and Construction (AEC) industry. Heiskanen (2017) suggested that when Blockchain is combined with IoT, the combination will allow the construction industry to achieve more trust in the quality of products. Turk and Klinc (2017) and Mathews et al. (2017) suggested that the Blockchain can generate a trustworthy environment, where the stakeholders share their BIM data. Finally, Li et al. (2018) investigated the challenges and opportunities from the distributed ledger technology of Blockchain in the built environment. Although all these studies have investigated the potential of Blockchain to improve construction industry, few studies have attempted to provide detailed scenarios for Blockchain use cases. In addition, they have not yet verified the technical feasibility of the proposed ideas, which utilize the Blockchain technology, or validate its potential. In this research, we provide three specific scenarios regarding Blockchain application in CSC. Furthermore, through an expert interview, we verify the technical feasibility of the scenarios with Blockchain-based software developers and validate its potential with the construction industry professionals.

\section{Three Scenarios of Blockchain-based Information Sharing in CSC}

\section{Scenario 1 Reliable Information Sharing}

Due to concerns about data security and potential misuse of data, stakeholders in construction projects may be reluctant to use information-sharing platforms, even though it increases both the project value and the stakeholders' profits. These concerns about mistrust are associated with the risk of data falsification or tampering for enhancing individual profits or avoiding liabilities. Kerschbaum and Chaves (2011) suggest that in a supply chain, suppliers are reluctant to share item-level data because business critical information might be inferred and taken advantage of from these data. Assume that one stakeholder manages the information-sharing platform. How can other stakeholders ensure that the party who is managing the platform is trustworthy and would not falsify or tamper with data for 
their individual business interests? For example, if a GC manages the information-sharing platform and collects as well as stores all different Building Information Modeling (BIM) Level of Development (LOD) submissions from A/E throughout different phases of the project, submittals, change orders, and all materials' data on quality, quantity, and delivery time from Subs. When legal issues on project delay, cost, or quality arise, an unethical GC could potentially take advantage of or even falsify the data for their own individual interests to avoid potential liabilities. Given this situation, the parties may be hesitant to share their critical data to protect themselves from the potentially opportunistic behavior of others (Dossick \& Neff, 2009). Let's imagine a second scenario where a third-party entity manages the information-sharing platform. In this case, how can the stakeholders ensure that the data would not be tampered with or falsified by the third-party entity or any other stakeholders? Other concerns could include the risk of third-party entity going out of business or be attacked by hackers, and the risks of information leakage. If the information-sharing platform is based on Blockchain technology, the stakeholders will not be concerned about the possibility of data being falsified or tampered with by anyone. Blockchain provides a trust-enhanced environment where the exchanged data are stored in blocks, which are cryptographically hashed, and the blocks are linked together with this hash (See Figure 1). Once the data are modified, the hash will change, indicating that the data are no longer the same. The data with a different hash cannot be considered as correct data because the link with the previous blocks will be broken. Furthermore, all the transaction data in the Blockchain are replicated and shared across the entire network. It is thus practically impossible to falsify the data just by changing one stakeholder's data.

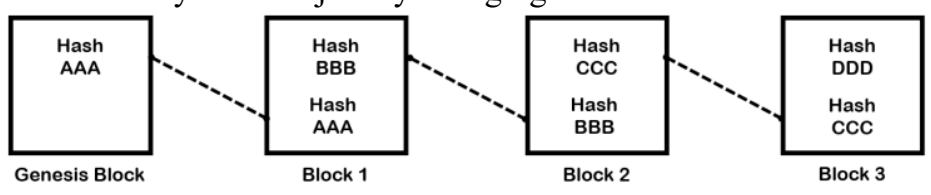

Figure 1. Linked Blocks by Hash

Blockchain-based information-sharing platform generates a decentralized environment, which enables peer-to-peer transactions (See Figure 2). In this platform, central storage is unnecessary, and the data transaction occur peer-to-peer. This peer-to-peer data transaction will encourage the stakeholders to share their information because it does not need a central authority such as a GC or any other thirdparty entity.

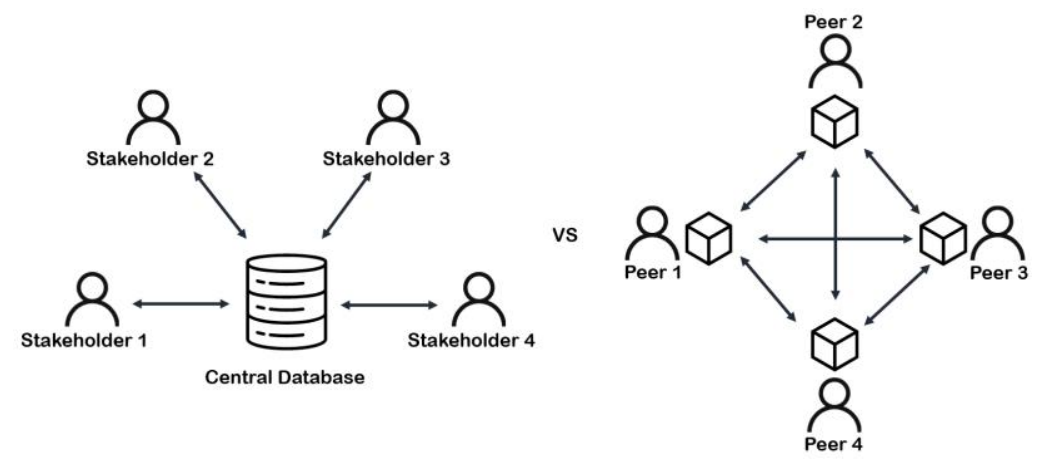

Figure 2. Central Database Transaction Versus Peer-to-peer Transaction

These above capabilities provide a trust-enhanced information-sharing platform and, thus, the stakeholders can share reliable information for both greater value of the project and their individual profits. For example, the trust-enhanced environment will reduce the number of change orders by inducing information sharing between $\mathrm{A} / \mathrm{E}$ and $\mathrm{GC}$ in early stages of the project; the reliable information sharing between GC and Subs will decrease the uncertainty of product delivery date, 
quality, and cost, leading to better performance on material management; owners will be able to trust the authenticity of all transactions-related information in the project.

\section{Scenario 2 Prevention on Counterfeiting, Fraudulent, Sub-Standard Material}

The non-repeating one-of-a-kind projects, high initial investments, long payback periods, and tight profit margins make the construction industry especially vulnerable to the counterfeiting, fraudulent, and sub-standard material (CFSM) (Naderpajouh et al., 2014). CFSM should be managed and mitigated as it negatively impacts the cost, time, safety, and quality of the construction projects (Minchin Jr et al., 2012). The fundamental enabling source for CFSM is information asymmetry leading to opportunistic behavior of suppliers (Engebø et al., 2017). For example, unethical Subs can have more information than GC on materials such as quality, cost, and origin of the material and they may conceal or falsify the information for their individual profits. Let's imagine that a sub-contractor is asked to make six tons of steel panels using " $A$ " brand steel with specific grade of Material Test Certificates (MTCs) including chemical composition and tension test. In this setting, Quality Metals (2018) introduced two types of potential counterfeiting. For example, the sub-contractor might use four tons of " $\mathrm{A}$ " brand and two tons of a cheaper or inferior "B" brand and sell all six tons of panels under the guise of "A" brand. Furthermore, the sub-contractor may falsify the results of MTCs because the quality of panels could not meet the qualification. If we were using a Blockchain-based information-sharing platform, such a situation can be prevented. In a Blockchain-based platform, all transactions of the sub-contractor will be recorded and shared with other stakeholders (See Figure 3). Furthermore, the if-this-then-that principle of smart contracts facilitates the fulfillment of the obligations according to the contracts without a third-party enforcer. For example, in a smart contract, if the conditions such as quality, quantity, or delivery-time of products are consistent with the contract, then the costs will be paid automatically. It will encourage suppliers to be committed to the product quality and delivery punctuality, and prevent the opportunistic behavior of suppliers. In this trust-enhanced environment, making and providing CFSM will be impossible.

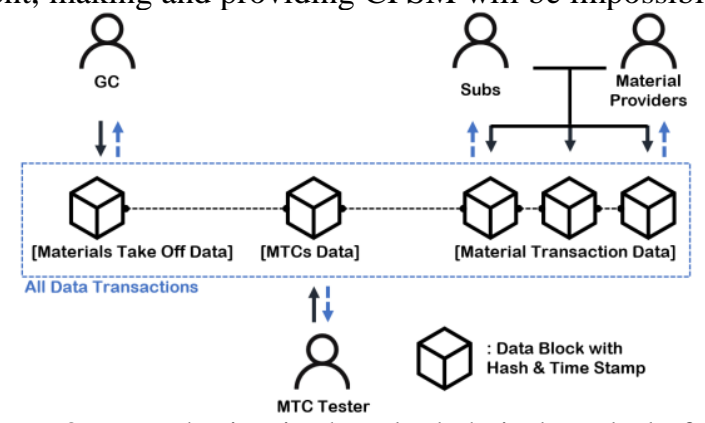

Figure 3. Data sharing in the Blockchain-based Platform

\section{Scenario 3 Reducing Bullwhip Effect in CSC}

Bullwhip Effect refers that a downstream demand shock, such as an increase in quantity of products ordered, is increasingly distorted and, thus, amplified as the transaction moves upstream in the supply chain (Lee et al., 2004). This effect leads to unnecessary inventory and backlog costs. For example, in CSC, materials quantity data of transaction between GC to Subs may be distorted and thus amplified in the transaction between component manufacturer and raw material supplier. The reason is that each stakeholder in the supply chain individually forecasts and plans material requirements due to lack of information sharing among all stakeholders (Taylor \& Bjornsson, 1999). According to Taylor and Bjornsson (1999), one of the reasons why stakeholders are reluctant to share all necessary information 
is that they don't trust one another regarding information-misuse and information-leakage. As explained in previous sections, Blockchain technology enables a trust-enhanced environment where information can be shared among all stakeholders with security through cryptographic encryption and the information is tamper-proof. It may encourage the stakeholders to share information in CSC so that the bullwhip effect is diminished.

\section{In-depth written interviews for validation}

Table 1

Scenario validation: Questions

\begin{tabular}{|c|c|}
\hline Interviewee & Questionnaire \\
\hline & $\begin{array}{l}\text { 1. The problems described in the scenarios are prevailing in the construction } \\
\text { industry and not have been addressed. }\end{array}$ \\
\hline $\begin{array}{l}\text { Construction } \\
\text { professionals }\end{array}$ & $\begin{array}{l}\text { 2. The root cause of the problems is the lack of trust resulted from insecure } \\
\text { information sharing, potential counterfeit, and difficulty of tracing the data } \\
\text { transaction history. }\end{array}$ \\
\hline & $\begin{array}{l}\text { 3. The benefits of Blockchain technology (e.g. cryptographically secured data } \\
\text { storage, tamper-proof, peer-to-peer data transaction) can effectively eliminate } \\
\text { the root cause of the lack of trust and facilitate secured information sharing. }\end{array}$ \\
\hline \multirow{2}{*}{$\begin{array}{l}\text { Blockchain } \\
\text { experts }\end{array}$} & $\begin{array}{l}\text { 1. In the scenarios, Blockchain is proposed as the most appropriate technology } \\
\text { to generate trust in information sharing by enabling a cryptographically secure } \\
\text { and tamper-proof storage for peer-to-peer data sharing. }\end{array}$ \\
\hline & $\begin{array}{l}\text { 2. The proposed Blockchain-based solutions described in the scenarios are } \\
\text { feasible and reasonable. }\end{array}$ \\
\hline
\end{tabular}

A questionnaire survey containing five questions was developed to validate the proposed ideas discussed in the scenarios and to verify their technical feasibility (See Table 1). The questionnaire regarding the potential effectiveness discussed in the scenarios was distributed to an architect and a construction professional who have over 20 years of experience in the construction industry. Both acknowledge that the problems described in the scenarios are prevailing and have not been addressed. The construction professional agrees that Blockchain can effectively reduce the root cause of the lack of trust and facilitate secure information sharing. However, the architect points out that even if Blockchain generates a tamper-proof database, the lack of trust among stakeholders will not be eliminated because distrust is related to how the information will be used, rather than if it will be counterfeited. The questionnaire regarding the technical feasibility was distributed to a Blockchainbased software development company (Flexsin Inc.) and a qualified technician who has over 20 years of experience in the construction industry. They agree that Blockchain is the most appropriate technology to generate trust in information sharing and thus it is a feasible and reasonable solution for the problems discussed in the scenarios.

\section{Conclusion}

In supply chain, information sharing is critical to facilitate decision making, reduce cost, accelerate schedule, and promote the final quality of the project. However, CSC has information flow that is not well integrated because it is fragmented in various stages or across numerous stakeholders. To improve this, previous studies focused on developing information-sharing platforms, but these studies did not address mechanisms for promoting trust for reliable information sharing. Even though several studies emphasized the importance of trust in sharing information, systematic solution that enforces or 
augments trust using technology was not developed. In this paper, we examine the potential of Blockchain technology as a solution for establishing a trust-enhanced environment through distributed ledger approach, enabling information sharing for CSC. We provide the three scenarios, which explore Blockchain applications in CSC, and the technical feasibility and the potential effectiveness of the Blockchain-based solution discussed in the scenarios are verified with the expert interview. In the interview, although the architect points out the lack of trust should be addressed with how the information is used, the construction professional agrees with the potential effectiveness to reduce counterfeiting and to facilitate reliable information sharing in CSC. The Blockchain experts verify the technical feasibility of the scenarios. These examinations will serve as a cornerstone for conducting future studies with greater depth and specificity.

Future research should validate whether the trust-enhanced environment in CSC could be established by Blockchain technology and whether the Blockchain-based information-sharing system could generate the benefits explained in these scenarios.

\section{References}

Barima, O. (2017). Leveraging the blockchain technology to improve construction value delivery: the opportunities, benefits and challenges. Construction Projects, 93-112.

Behera, P., Mohanty, R., \& Prakash, A. (2015). Understanding construction supply chain management. Production Planning \& Control, 26(16), 1332-1350.

Belle, I. (2017). The architecture, engineering and construction industry and blockchain technology. Digital Culture, 2017, 279-284.

Cheng, J. C., Law, K. H., Bjornsson, H., Jones, A., \& Sriram, R. (2010). A service oriented framework for construction supply chain integration. Automation in Construction, 19(2), 245-260.

Deng, Y., Gan, V. J., Das, M., Cheng, J. C., \& Anumba, C. (2019). Integrating 4D BIM and GIS for Construction Supply Chain Management. Journal of Construction Engineering and Management, 145(4), 04019016.

Dossick, C. S., \& Neff, G. (2009). Organizational divisions in BIM-enabled commercial construction. Journal of Construction Engineering and Management, 136(4), 459-467.

Engeb $\varnothing$, A., Kjesbu, N., Lædre, O., \& Lohne, J. (2017). Perceived Consequences of Counterfeit, Fraudulent and Sub-standard Construction Materials. Procedia engineering, 196, 343-350.

Fischer, M., Ashcraft, H. W., Reed, D., \& Khanzode, A. (2017). Integrating project delivery: Wiley Online Library.

Heiskanen, A. (2017). The technology of trust: How the Internet of Things and blockchain could usher in a new era of construction productivity. Construction Research and Innovation, 8(2), 66-70.

Irizarry, J., Karan, E. P., \& Jalaei, F. (2013). Integrating BIM and GIS to improve the visual monitoring of construction supply chain management. Automation in Construction, 31, 241254.

Jiang, Z., Henneberg, S. C., \& Naudé, P. (2012). Supplier relationship management in the construction industry: the effects of trust and dependence. Journal of Business \& Industrial Marketing.

Kerschbaum, F., \& Chaves, L. W. F. (2011). Secure sharing of item-level data in the cloud. Paper presented at the 2011 IEEE 4th International Conference on Cloud Computing.

Kim, M., \& Chai, S. (2017). The impact of supplier innovativeness, information sharing and strategic sourcing on improving supply chain agility: Global supply chain perspective. International Journal of Production Economics, 187, 42-52. 
Lee, H. L., Padmanabhan, V., \& Whang, S. (2004). Information distortion in a supply chain: the bullwhip effect. Management science, 50(12_supplement), 1875-1886.

Li, J., Greenwood, D., \& Kassem, M. (2018). Blockchain in the built environment: analysing current applications and developing an emergent framework.

Lisk. (2019). What is Blockchain? Retrieved from https://lisk.io/academy/blockchain-basics/what-isblockchain

Mathews, M., Robles, D., \& Bowe, B. (2017). BIM+ blockchain: A solution to the trust problem in collaboration?

Meng, X. (2010). Assessment framework for construction supply chain relationships: Development and evaluation. International journal of project management, 28(7), 695-707.

Minchin Jr, R. E., Cui, S., Walters, R. C., Issa, R., \& Pan, J. (2012). Sino-American opinions and perceptions of counterfeiting in the construction supply chain. Journal of Construction Engineering and Management, 139(1), 1-8.

Mougayar, W. (2016). The business blockchain: promise, practice, and application of the next Internet technology: John Wiley \& Sons.

Naderpajouh, N., Hastak, M., Gokhale, S., Bayraktar, M. E., Iyer, A., \& Arif, F. (2014). Counterfeiting risk governance in the capital projects supply chain. Journal of Construction Engineering and Management, 141(3), 04014084.

Nummelin, J., Sulankivi, K., Kiviniemi, M., \& Koppinen, T. (2011). Managing building information and client requirements in construction supply chain: constructor's view. Paper presented at the Conference Proceedings of CIB W078-W102 2011 joint conference. Sophia Antipolis, FR, 26-28 Oct. 2011.

Quality Metals, I. (2018). Get the Facts on Counterfeit Steel and How to Avoid it. Retrieved from https://www.qualitymetalsinc.com/get-facts-counterfeit-steel-avoid/

Rosic, A. (2016). Smart Contracts: The Blockchain Technology That Will Replace Lawyers. Retrieved from https://blockgeeks.com/guides/smart-contracts/

Ryciuk, U. (2017). Identification of factors related to trust formation in construction supply chains. Procedia engineering, 182, 627-634.

Saini, M., Arif, M., \& Kulonda, D. J. (2019). Challenges to transferring and sharing of tacit knowledge within a construction supply chain. Construction Innovation, 19(1), 15-33.

Samaniego, M., \& Deters, R. (2016). Blockchain as a Service for IoT. Paper presented at the 2016 IEEE International Conference on Internet of Things (iThings) and IEEE Green Computing and Communications (GreenCom) and IEEE Cyber, Physical and Social Computing (CPSCom) and IEEE Smart Data (SmartData).

Shin, T.-H., Chin, S., Yoon, S.-W., \& Kwon, S.-W. (2011). A service-oriented integrated information framework for RFID/WSN-based intelligent construction supply chain management. Automation in Construction, 20(6), 706-715.

Taylor, J., \& Bjornsson, H. (1999). Construction supply chain improvements through internet pooled procurement. Paper presented at the Proceedings of IGLC.

Turk, Ž., \& Klinc, R. (2017). Potentials of blockchain technology for construction management. Procedia engineering, 196, 638-645.

Wang, L.-C., Lin, Y.-C., \& Lin, P. H. (2007). Dynamic mobile RFID-based supply chain control and management system in construction. Advanced Engineering Informatics, 21(4), 377-390.

Xiang, P., Zhou, J., Zhou, X., \& Ye, K. (2012). Construction project risk management based on the view of asymmetric information. Journal of Construction Engineering and Management, 138(11), 1303-1311. 